\title{
Short-term survivors in glioblastomas with oligodendroglioma component: a clinical study of 186 Chinese patients from a single institution
}

\author{
Haihui Jiang $\cdot$ Xiaohui Ren $\cdot$ Junmei Wang $\cdot$ \\ Zhe Zhang $\cdot$ Wenqing Jia $\cdot$ Song Lin
}

Received: 18 April 2013/Accepted: 10 November 2013/Published online: 22 November 2013

(C) The Author(s) 2013. This article is published with open access at Springerlink.com

\begin{abstract}
This study was designed to display the molecular genetic features of short-term survivors in glioblastomas with oligodendroglioma component (GBMO). A total of 186 patients with histological diagnosis of primary gliomas, including 11 GBMO-STS (short-term survivors, survival $\leq 12$ months), 29 GBMO-LTS (relatively longterm survivors, survival $>12$ months), 36 anaplastic oligoastrocytoma (AOA) and 110 glioblastoma multiforme (GBM), enrolled in the study. An evaluation form was developed and used to document molecular pathological, clinical and treatment-associated parameters between subgroups. Kaplan-Meier plots for survival showed that the median progression-free survival (PFS) and overall survival (OS) of GBMO-STS were 5.0 and 10.0 months, respectively. Intergroup comparison revealed that the GBMO-STS harbored the most dismal prognosis than those with AOA, GBMO-LTS or GBM $(P<0.001$ for PFS, $P<0.001$ for OS, respectively). Cox regression analyses revealed that $1 \mathrm{p} / 19 \mathrm{q}$ co-deletion and $19 \mathrm{p}$ polysomy were independent prognostic factors $(P<0.05)$. Pearson's Chi square test demonstrated GBMO-STS exhibited lower $1 \mathrm{p} /$ $19 \mathrm{q}$ co-deletion, IDH1 mutation rates than AOA or GBMO-LTS $(P=0.032, P=0.045$ for $1 \mathrm{p} / 19 \mathrm{q}$ co-deletion; $P=0.034, \quad P=0.005$ for IDH1 mutation,
\end{abstract}

Electronic supplementary material The online version of this article (doi:10.1007/s11060-013-1311-3) contains supplementary material, which is available to authorized users.

H. Jiang $\cdot$ X. Ren $\cdot$ Z. Zhang $\cdot$ W. Jia $\cdot$ S. Lin $(\square)$

Neurosurgery, Beijing Tiantan Hospital, Capital Medical

University, Beijing 100050, China

e-mail: linsong2005@126.com

\section{J. Wang}

Pathology, Beijing Neurosurgical Institute, Capital Medical

University, Beijing 100050, China respectively) but higher chromosome 1q, 19p polysomy rates compared with AOA or GBM $(P=0.037, P=0.030$ for 1q polysomy; $P=0.017, P=0.011$ for 19 p polysomy, respectively). Patients with glioblastomas with oligodendroglioma component concurrent with polysomy for chromosomes 1 and 19 always confers an unfavorable prognosis which needs our extra attention in clinic.

Keywords GBMO $\cdot$ Short-term survivors ·

Polysomy

\section{Introduction}

The latest WHO classification of tumors of central nervous system (CNS) introduced an entity-AOA with necrosis was to be diagnosed as glioblastomas with oligodendroglioma component (GBMO). As a new pathology entity, the studies devoted to revealing the prognosis of GBMO were relatively limited.

Increasing evidence suggested GBMO was a heterogeneous group with considerable survival variant. Several reports found that the survival of GBMO was significantly longer than glioblastoma multiforme (GBM) whose median overall survival (OS) was only 12-15 months in spite of multimodal aggressive treatment, comprising surgical resection, local radiotherapy and systemic chemotherapy [1-3]. Nevertheless, reports showed GBMO without significant prognosis advantage over GBM were also consecutively published in the latest years [4-6]. In the present study, we found a small fraction of patients who were formerly diagnosed with AOA but displayed OS $\leq 12$ months. However, after the pathology re-evaluation, all these patients were confirmed as AOA with necrosis 
which should be classified into GBMO according to the latest WHO classification of CNS tumors.

GBMO displayed OS $\leq 12.0$ months is a relatively rare event which is worthy of further inquiry. It has not been firmly established which, if any, of the molecular genetic aberrations is important for the pathogenesis of GBMOSTS represent prognostic factors. The identification of molecular genetic markers that are associated with survival in patients with GBMO would be beneficial for its diagnostic and prognostic potential. In this regard, we report a retrospective analysis of 186 primary high-grade gliomas recruited in the Beijing Tiantan Hospital Neurosurgery Department. In addition to basic clinical data, we evaluate the clinical characteristics and screen for glioma associated genetic aberrations, i.e. $1 \mathrm{p} / 19 \mathrm{q}$ co-deletion, IDH1 mutation, MGMT promoter methylation, PTEN, p53, Ki-67, EGFR, VEGF expression, as well as chromosome 1q, 19p polysomy.

\section{Materials and methods}

\section{Patients}

A total of 186 patients (male 111 and female 75) with histological diagnosis of primary supratentorial high-grade gliomas (including 36 AOA, 11 GBMO-STS, 29 GBMOLTS and 110 GBM) in Beijing Tiantan Hospital from May 2008 to May 2011 were enrolled in the study. The secondary GBM were excluded. All patients provided written informed consent for the current study and the clinical study was approved by the Medical Ethics Committee of Capital Medical University. The mean age of this cohort was $47.5 \pm 12.5$ years at the time of surgery. All specimens were independently re-evaluated by three experienced neuro-pathologists according to the 2007 WHO classification of the CNS tumors [7]. In case of a discrepancy, the three observers simultaneously reviewed the slides to achieve a consensus. Patients who underwent needle biopsies prior resection, and/or prior adjuvant therapy (radiotherapy or chemotherapy) were excluded. These were done to create a more uniform patient population which could be propitious to the study.

\section{Treatment}

All the patients with gliomas, in our institution, were treated according to the National Comprehensive Cancer Network (NCCN) guideline. Patients, in our department, once pathologically diagnosed with high grade glioma, systemic chemotherapy and radiotherapy will be attempted after operation. Maximal tumor bulk resection while preserving the vital eloquent cortex was the principle goal during operation. Intraoperative subcortical electrical stimulation was performed when necessary. Extent of resection was assessed by the intraoperative ultrasound. Postoperative radiotherapy was routinely delivered to patients within 1 month after operation. The total dose was $60 \mathrm{~Gy}$, which was divided into 30 daily fractions of $2 \mathrm{~Gy}$ each. Meanwhile, postoperative chemotherapy was given; the common course of chemotherapy was 4-6 cycles which depended on the tolerance of toxic effect. The adjuvant chemotherapy drugs were mainly nimustine (ACNU) or temozolomide (TMZ).

\section{Recorded variables}

The clinical, operative, and hospital course records of 186 patients who met the inclusion criteria were retrospectively reviewed. The following information was recorded including patient's age, gender, removal degree, location of tumor, adjuvant therapy, and molecular parameters. The molecular parameters in this study included 1p/19q, IDH1, MGMT, PTEN, p53, Ki-67, EGFR, and VEGF. The status of chromosomes 1 and 19 was detected by fluorescence in situ hybridization (FISH) method, and IDH1 was sequenced. PTEN, p53, Ki-67, EGFR, VEGF expression were detected by immunohistochemical method. The MGMT promoter methylation was analyzed by methylation-specific PCR (MSP).

Assessment of $1 \mathrm{p} / 19 \mathrm{q}$ status by the fluorescence in situ hybridization (FISH) method

$1 p / 19 q$ co-deletion was detected by FISH method as described previously [8]. Tumors with more than $30 \%$ of nuclei showing DNA loss were defined as tumor with chromosomal loss. The tumor was considered to have polysomy if $>30 \%$ of nuclei showed more than two 1q and $19 \mathrm{p}$ signals.

\section{IDH1 sequence analysis}

Genomic DNA was isolated from snap-frozen tissue using the QIAmp DNA mini-kit, as described by the manufacturer (Qiagen). A fragment of 254 bp length spanning the catalytic domain of IDH1 including codon 132 was amplified using the sense primer IDH1 F: 5'-ACCAAAT GGCACCATACG- $3^{\prime}$ and the antisense primer IDH1 R: 5'-TTCATACCTTGCTTAATGGGG-3'. PCR using standard buffer conditions, $30 \mathrm{ng}$ of DNA and GoTaq DNA Polymerase (TaKaRa, Japan) employed 35 cycles with denaturing at $95{ }^{\circ} \mathrm{C}$ for $30 \mathrm{~s}$, annealing at $54{ }^{\circ} \mathrm{C}$ for $45 \mathrm{~s}$ and extension at $72{ }^{\circ} \mathrm{C}$ for $50 \mathrm{~s}$ in a total volume of $25 \mu \mathrm{L}$. The PCR amplification product was sent to Beijing Tianyi 
Huiyuan Bioscience and Technology Incorporation for sequencing.

Immunohistochemical analysis

Evaluation of PTEN, p53, Ki-67, EGFR, VEGF was detected by immunohistochemistry (IHC) as described previously [9]. The expression levels were based on the percentage of immunopositive cells (negative $<10 \%$ of tumor cells; positive $\geq 10 \%$ of tumor cells) (Table S1).

MGMT promoter methylation analysis

Genomic DNA was isolated from frozen tumor tissue by using Qiagen kit (Qiagen, Valencia, CA). MGMT promoter methylation was analyzed by MSP. Tumor DNA $(2 \mu \mathrm{g})$ was treated with sodium bisulfite using the $\mathrm{CpG}$ genome DNA modification kit (Qiagen). The primer sequences for the unmethylated reaction were $5^{\prime}$-TTTGTGTTTTGATGTT TGTAGGTTTTTGT- $3^{\prime}$ (forward) and 5'-AACTCCACACT CTTCCAAAAACAAAACA- $3^{\prime}$ (reverse). For the methylated reaction, they were $5^{\prime}$-TTTCGACGTTCGTAGGTTT TCGC- $3^{\prime}$ (forward) and $5^{\prime}$-GCACTCTTCCGAAAACG AAACG- $3^{\prime}$ (reverse). The annealing temperature was $59{ }^{\circ} \mathrm{C}$. The PCR products were separated on $4 \%$ agarose gels. The investigators who selected and analyzed the glioblasoma samples were blinded to all clinical information. Pyrosequencing analysis was carried out by Gene Tech (Shanghai) Company Limited. The GBM samples [methylation values $(10 \%)$ ] were considered as being methylated.

\section{Follow-up}

The progression-free survival (PFS) was designated as the time period from the first operation to the time of tumor recurrence or evidence of progression based on magnetic resonance imaging (MRI). Patients who were recurrencefree at last follow-up were considered as a censored event in analysis. OS was defined as the period between the first operation and death or last follow-up. Patients who were still alive at last follow-up were considered as a censored event in analysis. All the survival data were collected mainly when patients visited the clinics and during the phone interview with patients and/or their relatives.

\section{Statistical analysis}

SPSS 13.0 (SPSS for Windows, version 13.0 [SPSS Inc., Chicago, IL, USA]) was used for statistical analysis. Pearson's Chi square test and Fisher's exact test were used to compare the frequencies between groups. Kaplan-Meier method was used for survival analysis. Probability value was obtained from two-sided tests, with a statistical significance of $P<0.05$.

\section{Results}

Basic characteristics

The basic clinical characteristics of these patients enrolled in the study were summarized in Table 1. A total of 186 patients with primary high-grade gliomas who were surgically treated in our institution met the inclusion criteria. There were 111 male and 75 female with a mean age of $47.5 \pm 12.5$ years old, including $36(19.4 \%)$ AOA, 11 $(5.9 \%)$ GBMO-STS, 29 (15.6\%) GBMO-LTS and 110 $(59.1 \%)$ GBM. The median follow-up period of the 186 patients was 13.5 months (range 1.0-42.0 months). A total number of 94 patients had dead.

\section{Survival analyses of AOA, GBMO-STS, GBMO-LTS and GBM}

In the cohort 186 high-grade glioma, univariate analysis demonstrated that $1 \mathrm{p} / 19 \mathrm{q}$ co-deletion, polysomy for $1 \mathrm{q}$ and 19 p were associated with prognosis $(P<0.05)$ (Fig. S1). In multivariate Cox regression analysis, the presence of $1 \mathrm{p} / 19 \mathrm{q}$ co-deletion and $19 \mathrm{p}$ polysomy were independent prognostic factors $(P<0.05)$ (Table 2$)$. Unexpectedly, we haven't found the prognostic value of MGMT promoter methylation, PTEN, p53, Ki-67, EGFR, VEGF expression $(P>0.05)$.

The median PFS and OS of GBMO-STS were 5.0 [95 \% CI 3.382-6.618] and 10.0 [95 \% CI 7.977-12.023] months, respectively, which were significantly shorter than AOA, GBMO-LTS or GBM $(P<0.001$ for PFS, $P<0.001$ for OS, respectively) (Fig. 1 and Table S2).

According to the status of chromosomes 1 and 19 (codeletion yes or no, and polysomy yes or no), we could classified the 186 patients into four subgroups (subgroup 1, without co-deletion or polysomy; subgroup 2, with codeletion but without polysomy; subgroup 3, with polysomy but without co-deletion; subgroup 4 , with polysomy and co-deletion) which conferred different survival time. Patients in subgroup 2 exhibited the most favorable prognosis compared with subgroup 1,3 and $4(P<0.001$ for PFS, $P=0.002$ for OS, respectively). Patients in subgroup 3 had the shortest survival time that the median PFS was 11.0 months and the OS was only 17.5 months. No significant difference of prognosis was observed between subgroup 1 and $4(P=0.803$ for PFS, $P=0.868$ for OS, respectively) (Fig. 2). 
Table 1 Clinical characteristics of AOA, GBMO-STS, GBMO-LTS, and GBM

\begin{tabular}{|c|c|c|c|c|c|c|c|c|c|}
\hline \multirow[t]{2}{*}{ Characteristic } & \multicolumn{2}{|l|}{ Subgroup 1} & \multirow[t]{2}{*}{$P$ value } & \multicolumn{2}{|l|}{ Subgroup 2} & \multirow[t]{2}{*}{$P$ value } & \multicolumn{2}{|l|}{ Subgroup 3} & \multirow[t]{2}{*}{$P$ value } \\
\hline & $\begin{array}{l}\text { GBMO-STS } \\
(n=11)\end{array}$ & $\begin{array}{l}\text { AOA } \\
(n=36)\end{array}$ & & $\begin{array}{l}\text { GBM } \\
(n=110)\end{array}$ & $\begin{array}{l}\text { GBMO-STS } \\
(n=11)\end{array}$ & & $\begin{array}{l}\text { GBMO-LTS } \\
(n=29)\end{array}$ & $\begin{array}{l}\text { GBMO-STS } \\
(n=11)\end{array}$ & \\
\hline \multicolumn{10}{|l|}{ Age (years) } \\
\hline Median (range) & $41.0(14-58)$ & $44.0(30-68)$ & 0.157 & $52.0(12-70)$ & $41.0(14-58)$ & $0.048 *$ & $43.0(17-59)$ & $41.0(14-58)$ & 0.348 \\
\hline \multicolumn{10}{|l|}{ Gender } \\
\hline Male (\%) & $6(54.5)$ & $18(50.0)$ & & $70(63.6)$ & $6(54.5)$ & & $17(58.6)$ & $6(54.5)$ & \\
\hline Female $(\%)$ & $5(45.5)$ & $18(50.0)$ & 0.792 & $40(36.4)$ & $5(45.5)$ & 0.789 & $12(41.4)$ & $5(45.5)$ & 1.0 \\
\hline \multicolumn{10}{|l|}{ Tumor location } \\
\hline Temporal (\%) & $4(36.4)$ & $9(25.0)$ & & $41(37.3)$ & $4(36.4)$ & & $10(34.5)$ & $4(36.4)$ & \\
\hline Frontal (\%) & $4(36.4)$ & $15(41.7)$ & & $37(33.6)$ & $4(36.4)$ & & $11(37.9)$ & $4(36.4)$ & \\
\hline Parietal (\%) & $2(18.1)$ & $5(13.9)$ & & $17(15.5)$ & $2(18.1)$ & & $4(13.8)$ & $2(18.1)$ & \\
\hline Occipital (\%) & $0(0.0)$ & $1(2.8)$ & & $4(3.6)$ & $0(0.0)$ & & $0(0.0)$ & $0(0.0)$ & \\
\hline Insular (\%) & $1(9.1)$ & $3(8.3)$ & & $6(5.5)$ & $1(9.1)$ & & $4(13.8)$ & $1(9.1)$ & \\
\hline Others $(\%)$ & $0(0.0)$ & $3(8.3)$ & $>0.05$ & $5(4.5)$ & $0(0.0)$ & $>0.05$ & $0(0.0)$ & $0(0.0)$ & $>0.05$ \\
\hline \multicolumn{10}{|c|}{ Preoperative epilepsy } \\
\hline Yes $(\%)$ & $3(27.3)$ & $13(36.1)$ & 0.859 & $25(22.7)$ & $3(27.3)$ & 1.0 & $5(20.7)$ & $3(27.3)$ & 0.791 \\
\hline \multicolumn{10}{|l|}{ Preoperative KPS } \\
\hline Median (range) & $80(60-90)$ & $75(50-100)$ & 0.768 & $80(50-100)$ & $80(60-90)$ & 0.369 & $80(60-100)$ & $80(60-90)$ & 0.677 \\
\hline \multicolumn{10}{|l|}{ Tumor resection } \\
\hline GTR (\%) & $6(54.5)$ & $25(69.4)$ & 0.583 & $52(47.3)$ & $6(54.5)$ & 0.645 & $18(62.1)$ & $6(54.5)$ & 0.942 \\
\hline \multicolumn{10}{|l|}{ Nimustine } \\
\hline Yes $(\%)$ & $11(100.0)$ & $34(94.4)$ & $1.0^{\#}$ & $106(96.4)$ & $11(100.0)$ & $1.0^{\#}$ & $26(89.7)$ & $11(100.0)$ & $0.548^{\#}$ \\
\hline \multicolumn{10}{|l|}{ Radiotherapy } \\
\hline Yes $(\%)$ & $11(100.0)$ & 33 (91.7) & $1.0^{\#}$ & $102(92.7)$ & $11(100.0)$ & $1.0^{\#}$ & $28(96.6)$ & $11(100.0)$ & $1.0^{\#}$ \\
\hline \multicolumn{10}{|l|}{ Temozolomide } \\
\hline Yes $(\%)$ & $7(63.6)$ & $11(30.6)$ & 0.105 & $59(53.6)$ & 7 (63.6) & 0.525 & $21(72.4)$ & 7 (63.6) & 0.877 \\
\hline
\end{tabular}

KPS Karnofsky performance score, GTR gross-total resection

\# Fisher's exact test

$* P<0.05$

Table 2 Univariate and multivariate associations with survival for patients with highgrade gliomas

\begin{tabular}{lllll}
\hline Parameter & $\begin{array}{l}\text { Univariate analysis } \\
\text { Median survival } \\
(95 \% \mathrm{CI}) \text { (months) }\end{array}$ & $P$ value & $\begin{array}{l}\text { Multivariate analysis } \\
\text { OR }(95 \% \mathrm{CI})\end{array}$ & $P$ value \\
& & & \\
\hline
\end{tabular}

Factors associated with PFS

\begin{tabular}{llrlr} 
1p/19q co-deletion & N/A & $<0.001$ & $0.336(0.176-0.643)$ & 0.001 \\
1q polysomy & $9.0(3.452-14.548)$ & 0.003 & - & \\
19p polysomy & $7.0(4.798-9.202)$ & $<0.001$ & $2.575(1.608-4.124)$ & $<0.001$ \\
Factors associated with OS & & & \\
1p/19q co-deletion & N/A & 0.003 & $0.319(0.134-0.760)$ & 0.010 \\
1q polysomy & $17.5(13.404-21.596)$ & 0.056 & - & \\
19p polysomy & $17.0(13.835-20.165)$ & 0.009 & $1.930(1.064-3.502)$ & 0.031 \\
\hline
\end{tabular}

CI confidence interval

them, $28(23.1 \%)$ had 1p/19q co-deletion (including 14

Frequencies of $1 \mathrm{p} / 19 \mathrm{q}$ co-deletion, IDH1 mutation and 1q, 19p polysomy in AOA, GBMO-STS, GBMOLTS and GBM

Fluorescence in situ hybridization (FISH) for chromosome $1 \mathrm{p}$ and $19 \mathrm{q}$ was available in 121 cases. Among in AOA, 0 in GBMO-STS, 11 in GBMO-LTS and 3 in GBM). The frequencies of $1 p / 19 q$ co-deletion in AOA, GBMO-STS, GBMO-LTS and GBM were 40.0, $0.0 \%$, 37.9 and $6.5 \%$, respectively. The frequency of $1 p / 19 q$ co-deletion in GBMO-STS was significantly lower than 

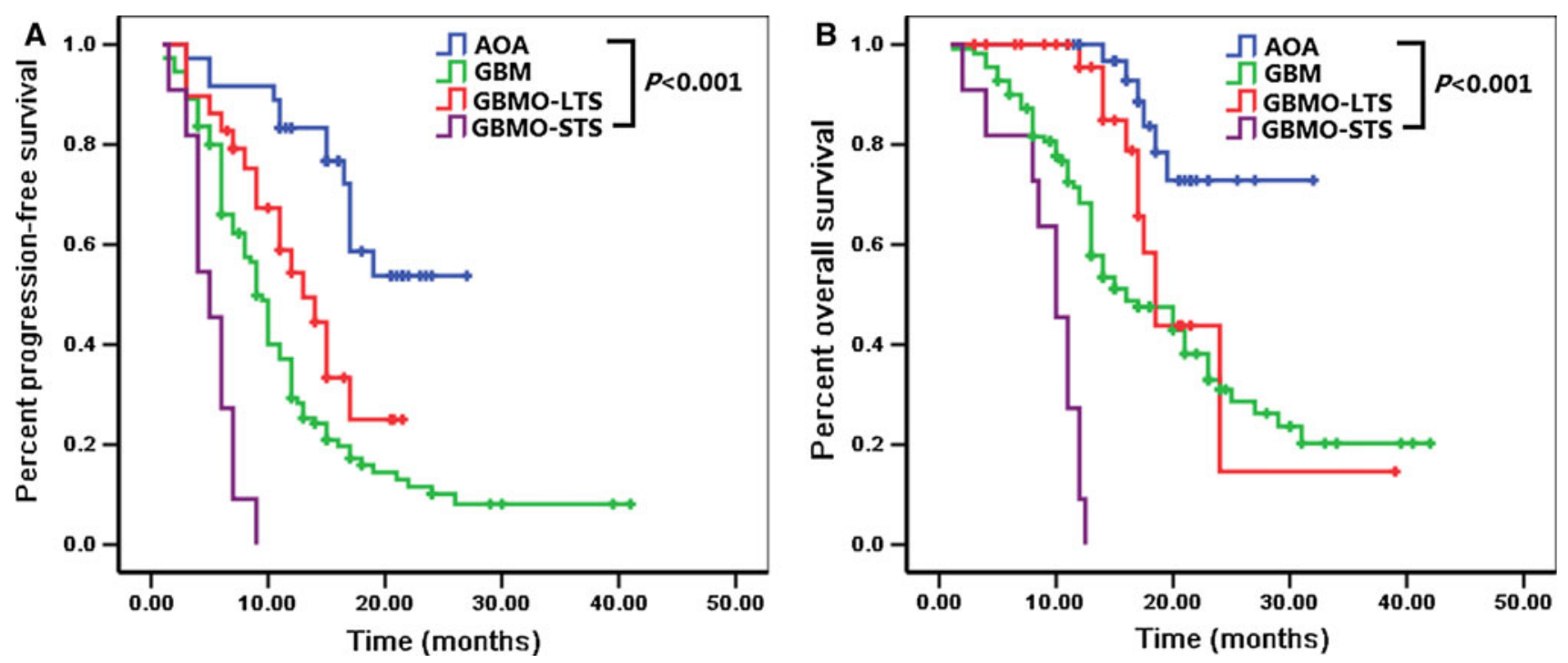

Fig. 1 Kaplan-Meier plots for PFS and OS between AOA, GBMO-STS, GBMO-LTS and GBM were significantly different $(P<0.001$ for PFS, $P<0.001$ for OS, respectively)

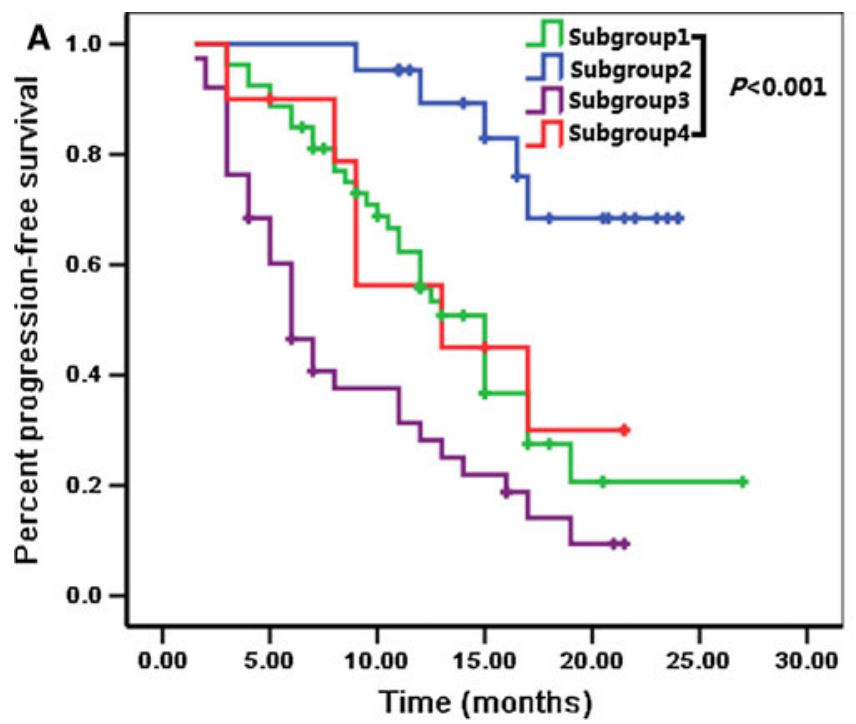

Fig. 2 Subgroup 1, without co-deletion or polysomy; Subgroup 2, with co-deletion but without polysomy; Subgroup 3, with polysomy but without co-deletion; Subgroup 4, with polysomy and co-deletion. The survival time of subgroup 1 and 4 was significantly shorter compared with subgroup $2(P=0.001$ for PFS, $P=0.015$ for OS,

those in AOA or GBMO-LTS $(P=0.032$ and $P=0.045$, respectively).

DNA sequencing for IDH1 was available in 137 patients. Among them, 38 out of $137(27.7 \%)$ had IDH1 mutation (including 14 in AOA, 0 in GBMO-STS, 16 in GBMO-LTS and 8 in GBM). The frequencies of IDH1 mutation in AOA, GBMO-STS, GBMO-LTS and GBM were $42.4,0.0,55.2$ and $12.3 \%$, respectively. The

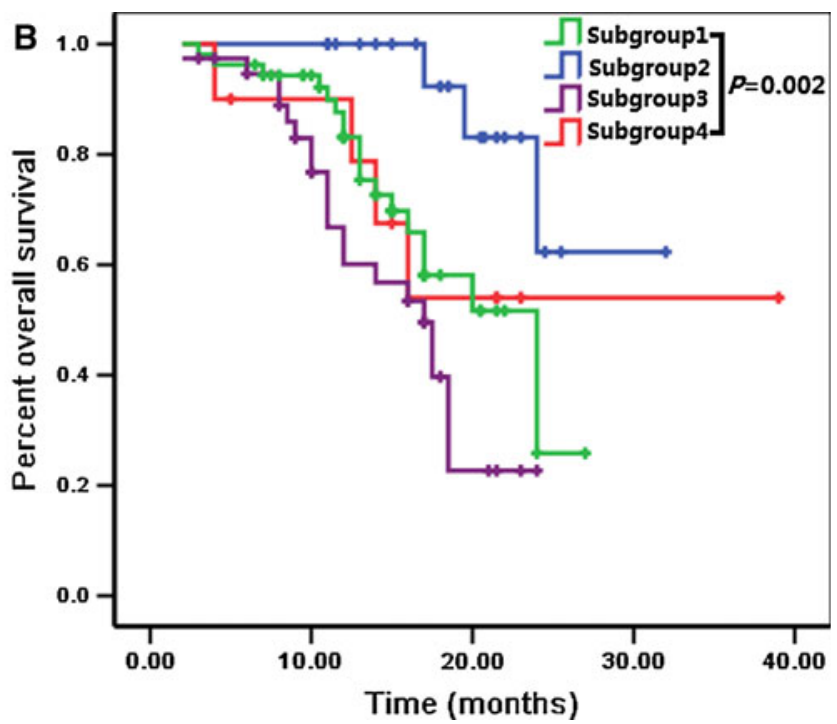

respectively) but longer than subgroup $3(P=0.001$ for PFS, $P=0.05$ for OS, respectively). No significant difference of prognosis was observed between subgroup 1 and $4(P=0.803$ for PFS, $P=0.868$ for OS, respectively)

frequency of IDH1 mutation in GBMO-STS was significantly lower than those in AOA or GBMO-LTS ( $P=0.034$ and $P=0.005$, respectively).

Chromosome polysomy status could be assessed in 123 patients. Of the 123 patients, $40(32.5 \%)$ had 1q ploysomy (including 8 in AOA, 7 in GBMO-STS, 13 in GBMO-LTS and 12 in GBM); 46 (37.4\%) had 19p ploysomy (including 9 in AOA, 8 in GBMO-STS, 16 in 


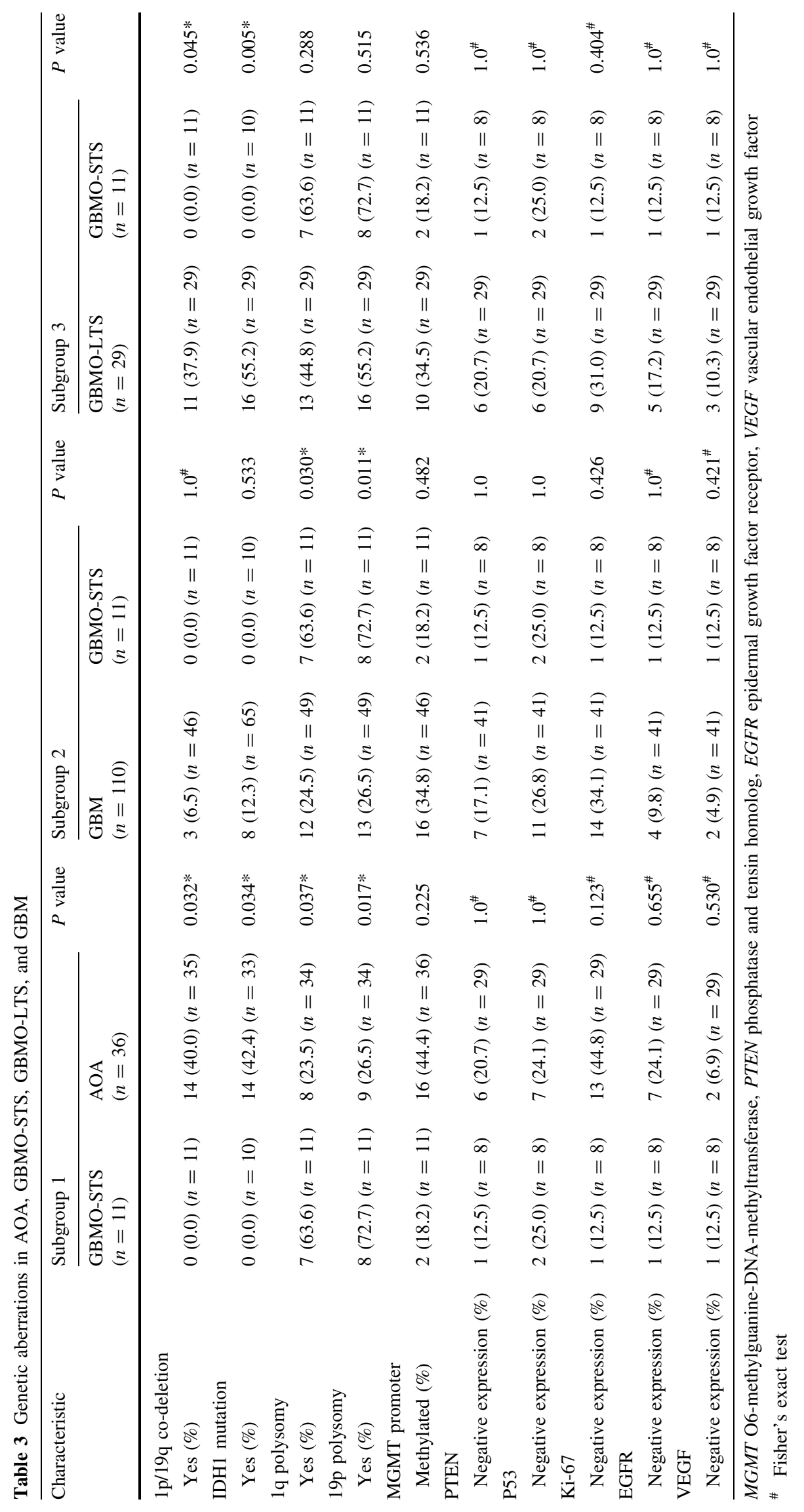


Table 4 The outcomes of selected series of GBMO and GBM

\begin{tabular}{|c|c|c|c|c|c|}
\hline Author & Patient group & $\begin{array}{l}\text { Number of } \\
\text { patients }\end{array}$ & Treatment & $\begin{array}{l}\text { Median OS } \\
\text { (months) }\end{array}$ & $P$ value \\
\hline \multirow[t]{2}{*}{ Miller et al. [2] } & AOA with necrosis & 71 & N/A & 22.8 & \\
\hline & GBM & 581 & N/A & 9.8 & $<0.0001 *$ \\
\hline Vordermark et al. [1] & GBMO & 10 & Post-OPT RT in $90 \%+$ ACNU and VM26 in $80 \%$ & 26.0 & N/A \\
\hline \multirow[t]{2}{*}{ Kanno et al. [3] } & GBMO & 17 & Post-OPT RT + ACNU + TMZ & $\approx 40.0^{\mathrm{a}}$ & \\
\hline & GBM & 52 & Post-OPT RT + ACNU + TMZ & $\approx 18.0^{\mathrm{a}}$ & 0.068 \\
\hline \multirow[t]{2}{*}{ Jiang et al. [10] } & GBMO & 40 & Post-OPT RT in $85 \%$; RT + Chemo in $62 \%$ & 19.0 & \\
\hline & GBM & 179 & Post-OPT RT in $87 \%$; RT + Chemo in $65 \%$ & 13.2 & $0.022 *$ \\
\hline \multirow[t]{2}{*}{ Pinto et al. [4] } & GBMO & 24 & Post-OPT RT + Chemo & 14.9 & \\
\hline & GBM & 64 & Post-OPT RT + Chemo & 13.5 & 0.566 \\
\hline \multirow[t]{2}{*}{ Hegi et al. [5] } & GBMO & 52 & Post-OPT RT + Concomitant TMZ + Ajuvand TMZ & N/A & \\
\hline & GBM & 287 & Post-OPT RT + Concomitant TMZ + Ajuvand TMZ & N/A & 0.48 \\
\hline Nakamura et al. [6] & GBMO & 19 & Post-OPT RT in $100 \%+$ ACNU/TMZ in $89.5 \%$ & 14.0 & N/A \\
\hline \multirow[t]{3}{*}{ Present study } & GBMO-STS & 11 & $\begin{array}{l}\text { Post-OPT RT in } 100 \%+\text { ACNU } \\
\text { in } 100 \%+\text { TMZ in } 63.6 \%\end{array}$ & 10.0 & \\
\hline & GBMO-LTS & 29 & $\begin{array}{l}\text { Post-OPT RT in } 96.6 \%+\text { ACNU } \\
\text { in } 89.7 \%+\text { TMZ in } 72.4 \%\end{array}$ & 18.5 & \\
\hline & GBM & 110 & $\begin{array}{l}\text { Post-OPT RT in } 92.7 \%+\text { ACNU } \\
\text { in } 96.4 \%+\mathrm{TMZ} \text { in } 53.6 \%\end{array}$ & 16.0 & $<0.001 *$ \\
\hline
\end{tabular}

${ }^{\text {a }}$ Estimated value from graph

$O P T$ operation, $R T$ radiation therapy, Chemo chemotherapy, $A C N U$ nimustine, $T M Z$ temozolomide, N/A not available

GBMO-LTS and 13 in GBM). 1q polysomy was slightly more frequent in GBMO-STS compared with AOA or GBM $(P=0.037$ and $P=0.030$, respectively). Furthermore, GBMO-STS exhibited higher $19 \mathrm{p}$ polysomy rate than AOA or GBM $(P=0.017$ and $P=0.011$, respectively) (Table 3).

\section{Discussion}

Anaplastic oligoastrocytoma (AOA) with necrosis, formerly categorized in WHO grade III, now is regarded as GBMO (WHO grade IV) which is a heterogeneous group with considerable survival variant. Several reports dedicated to the survival analysis about GBM versus GBMO showed that no favorable prognostic value of an oligodendroglial component was found [4-6], although some other studies indicated a longer survival for GBMO [1-3, 10] (Table 4). In the present study, we found a subtype of GBMO harbored shorter survival time compared with GBM. This is, so far as we know, the first series of GBMO short-term survivors reported to date. We provide a clinical characterization and report on molecular analyses of the 11 patients who have a survival time $\leq 12$ months in order to reveal the reasons for the dismal prognosis.
No significant difference in clinical characterization was found between AOA, GBMO-STS, GBMO-LTS and GBM

Considering variable prognostic factors such as the age of the patients, extent of resection, postoperative radiotherapy or chemotherapy, Karnofsky performance status (KPS) influenced the patients' survival, we recruited all the clinical factors in the present study. We found that except for the age at diagnosis of patients with GBM was older than those in GBMO-STS $(P=0.048)$, no other significant difference was observed between the four subgroups (AOA, GBMOSTS, GBMO-LTS and GBM) (Table 1). But the prognosis of patients with GBM was, unexpectedly, better than GBMOSTS. It suggested the prognostic value of age was covered after the adjustment for some potential prognostic factors, such as $1 \mathrm{p} / 19 \mathrm{q}$ co-deletion, polysomy for $1 \mathrm{q}$ and $19 \mathrm{p}$, in these high-grade gliomas.

High-frequency of $1 \mathrm{p} / 19 \mathrm{q}$ co-deletion and IDH1 mutation result in the survival advantage of AOA and GBMO-LTS

In our cohort, we found that the GBMO-STS exhibited lower frequency of $1 \mathrm{p} / 19 \mathrm{q}$ co-deletion than AOA or GBMO-LTS but resembled it with GBM. This finding 
was similar with Jiang's report, which maintained there was no significant difference of $1 p / 19 q$ co-deletion rate between GBMO and GBM [10]. 1p/19q co-deletion is an established genetic marker for prognostication about glioma patients' survival and chemosensitivity [11, 12]. In 1998, Cairncross and colleagues reported that loss of $1 \mathrm{p}$ (and $1 \mathrm{p} / 19 \mathrm{q}$ co-deletion) predicts a better response to procarbazine-lomustine-vincristine chemotherapy and a longer survival in patients with AO [13]. These findings have been reproduced in many subsequent studies, including prospective and randomized phase III trials $[14,15]$. Moreover, oligodendroglial tumors with loss of $1 \mathrm{p} / 19 \mathrm{q}$ showed a response to treatment with the alkylating drug TMZ and radiotherapy, indicating its predictive value for a broader spectrum of therapeutic regimens [16-18]. These results indicated that patients with AOA or GBMO-LTS had a significantly longer survival time than GBMO-STS might be linked to the higher incidence of $1 \mathrm{p} / 19 \mathrm{q}$ co-deletion.

As elaborated by experts, IDH1 mutation was associated with a better outcome in patients with low-grade diffuse gliomas, AA, GBM and had been shown to be a powerful independent prognostic factor for prolonged survival $[19,20]$. IDH1/2 genes encode for the cytosolic and mitochondrial nicotinamide adenine dinucleotide phosphate (NADPH)-dependent isocitrate dehydrogenase enzymes which play an vital role in the citric acid cycle. Wild-type IDH1/2 isozymes catalyze the oxidative carboxylation of isocitrate to a-ketoglutarate and reduce $\mathrm{NADP}+$ to NADPH during this process [21, 22]. Both the a-ketoglutarate and the released NADPH are known cell defenders against oxidative damage. Mutated IDH gene decreases the ability of the IDH enzyme to catalyze the conversion of isocitrate to a-ketoglutarate and leads to a decreased quantity of a-ketoglutarate and NADPH, making the cell more susceptible to oxidative stress [23]. In the present study, patients of GBMO-STS showed lower IDH1 mutation rate compared with AOA or GBMO-LTS, which suggested that the survival of GBMO-STS would be shorter than AOA or GBMO-LTS. However, the incidence of IDH1 mutation, in our cohort, was a little lower in comparison to the reports from Europe or America, but it was similar with Jiang's report which displayed the frequency of IDH1/2 mutation in Chinese AOA was $45.8 \%$. Ethnic differences might partly explain it [24-26]. Inevitably, the phenomenon might, to some extent, due to our imperfect experiment method. Of interest, there was no significant difference of IDH1 mutation rate between GBMO-STS and GBM. It documented that the incidence of IDH1 mutation could not be a parameter which resulted in the difference of survival time between the two groups.
Presence of $1 \mathrm{q}, 19 \mathrm{p}$ polysomy contributed to the dismal prognosis of GBMO-STS

Univariate analysis revealed that polysomy for $1 \mathrm{q}$ and $19 \mathrm{p}$ were associated with the dismal prognosis of patients with high-grade gliomas. These findings were in consistent with many previous reports. Snuderl et al. [27] reported that polysomy for chromosomes 1 and 19 predicted earlier recurrence in anaplastic oligodendrogliomas with concurrent $1 \mathrm{p} / 19 \mathrm{q}$ loss. Wiens et al. [28] documented that combined polysomy was associated with higher histological tumor grade and conferred poor survival likelihood. They concluded polysomy of $1 \mathrm{q}$ and/or $19 \mathrm{p}$ was a relatively frequent occurrence in oligodendrogliomas and usually conferred an unfavorable outcome. In the present study, intergroup comparison showed GBMO-STS harbored higher frequency of 1q, 19p polysomy than AOA or GBM. It might partly interpret the phenomenon that GBMO-STS with a median OS of merely 10.0 months which was significantly shorter than AOA or even GBM. Another possible explanation for the dismal prognosis of GBMO was that these tumors were in fact small cell GBM which exhibited shorter survival time than GBM and could mimic GBMO. Considering this issue, a pathology re-evaluation was performed. Though the pathology consultation result revealed these specimens were GBMO, the EGFR amplification information was the best means to making the distinction between small cell GBM and GBMO. Because of the limited experimental resource, the EGFR amplification was not available in our laboratory. Finally, higher incidence of polysomy for 1q and 19p might be a potential parameter which contributed to the survival time $\leq 12$ months of GBMO-STS.

From the above, experts, all over the world, in regard of the prognostic value of an oligodendrogial component in glioblastomas couldn't arrive at a consensus (Table 4). Based on the data displayed in the study, we speculate that GBMO, a heterogeneous group with considerable survival variant, directly being regarded as "glioblastomas" with relatively favorable outcome remains a subject needs further inquiry. Because there is a subtype GBMO with concurrent polysomy for chromosomes 1 and 19 exhibits shorter survival than GBM. When encountered with such subtype of patients, perhaps taking the polysomy for chromosomes 1 and 19 into account would be more reasonable in guiding the individual therapy in clinic care. Another important issue is that FISH seems to be more suitable for assessing loss of $1 p$ and $19 q$ compared with polymerase chain reaction (PCR) with regard to clinical significance of polysomy. Because, compared with PCR-based loss of heterozygosity (LOH) assays, additional polysomy information can be gleaned from the FISH analysis. 
Study limitation

The small sample of GBMO-STS which would weaken the conviction of this study to some extent. So we will enlarge our sample for the further inquiry in the future. It was also a limitation that the EGFR amplification information was absent in distinguishing GBMO from small cell GBM.

\section{Conclusions}

Patients with GBMO concurrent with polysomy for chromosomes 1 and 19 always confers an unfavorable prognosis which needs our extra attention in clinic.

Acknowledgments We acknowledge financial support by Beijing Natural Science Foundation (7122061). The author appreciates Dr. Lin Luo and Dr. Junmei Wang for pathology diagnosis of GBM, the pathology department, Beijing Neurosurgical Institute. Dr. Guang Li, Pathology department, Beijing Tiantan Hospital, Capital Medical University.

Financial support This work was supported by Beijing Natural Science Foundation (7122061).

Conflict of interest No potential conflicts of interest were disclosed.

Open Access This article is distributed under the terms of the Creative Commons Attribution License which permits any use, distribution, and reproduction in any medium, provided the original author(s) and the source are credited.

\section{References}

1. Vordermark D, Ruprecht K, Rieckmann P, Roggendorf W, Vince GH, Warmuth-Metz M, Kolbl O, Flentje M (2006) Glioblastoma multiforme with oligodendroglial component (GBMO): favorable outcome after post-operative radiotherapy and chemotherapy with nimustine (ACNU) and teniposide (VM26). BMC Cancer 6:247

2. Miller CR, Dunham CP, Scheithauer BW, Perry A (2006) Significance of necrosis in grading of oligodendroglial neoplasms: a clinicopathologic and genetic study of newly diagnosed highgrade gliomas. J Clin Oncol 24:5419-5426

3. Kanno H, Nishihara H, Narita T, Yamaguchi S, Kobayashi H, Tanino M, Kimura T, Terasaka S, Tanaka S (2012) Prognostic implication of histological oligodendroglial tumor component: clinicopathological analysis of 111 cases of malignant gliomas. PLoS One 7:e41669

4. Pinto LW, Araujo MB, Vettore AL, Wernersbach L, Leite AC, Chimelli LM, Soares FA (2008) Glioblastomas: correlation between oligodendroglial components, genetic abnormalities, and prognosis. Virchows Arch 452:481-490

5. Hegi ME, Janzer RC, Lambiv WL, Gorlia T, Kouwenhoven MC, Hartmann C, von Deimling A, Martinet D, Besuchet SN, Diserens AC, Hamou MF, Bady P, Weller M, van den Bent MJ, Mason WP, Mirimanoff RO, Stupp R, Mokhtari K, Wesseling P (2012) Presence of an oligodendroglioma-like component in newly diagnosed glioblastoma identifies a pathogenetically heterogeneous subgroup and lacks prognostic value: central pathology review of the EORTC_26981/NCIC_CE.3 trial. Acta Neuropathol 123:841-852

6. Nakamura H, Makino K, Kuratsu J (2011) Molecular and clinical analysis of glioblastoma with an oligodendroglial component (GBMO). Brain Tumor Pathol 28:185-190

7. Louis DN, Ohgaki H, Wiestler OD, Cavenee WK, Burger PC, Jouvet A, Scheithauer BW, Kleihues P (2007) The 2007 WHO classification of tumours of the central nervous system. Acta Neuropathol 114:97-109

8. Ren X, Cui X, Lin S, Wang J, Jiang Z, Sui D, Li J, Wang Z (2012) Co-deletion of chromosome $1 p / 19 q$ and IDH1/2 mutation in glioma subsets of brain tumors in Chinese patients. PLoS One $7: \mathrm{e} 32764$

9. Jiang H, Ren X, Zhang W, Ma J, Sui D, Jiang Z, Cui X, Lin S (2013) A new prognostic scoring scale for patients with primary WHO grade III gliomas based on molecular predictors. J Neurooncol 111:367-375

10. Wang Y, Li S, Chen L, You G, Bao Z, Yan W, Shi Z, Chen Y, Yao K, Zhang W, Kang C, Jiang T (2012) Glioblastoma with an oligodendroglioma component: distinct clinical behavior, genetic alterations, and outcome. Neuro Oncol 14:518-525

11. Jeuken JW, von Deimling A, Wesseling P (2004) Molecular pathogenesis of oligodendroglial tumors. J Neurooncol 70: $161-181$

12. Cairncross G, Jenkins R (2008) Gliomas with $1 p / 19 q$ codeletion: a.k.a. oligodendroglioma. Cancer J 14:352-357

13. Cairncross JG, Ueki K, Zlatescu MC, Lisle DK, Finkelstein DM, Hammond RR, Silver JS, Stark PC, Macdonald DR, Ino Y, Ramsay DA, Louis DN (1998) Specific genetic predictors of chemotherapeutic response and survival in patients with anaplastic oligodendrogliomas. J Natl Cancer Inst 90:1473-1479

14. Cairncross G, Berkey B, Shaw E, Jenkins R, Scheithauer B, Brachman D, Buckner J, Fink K, Souhami L, Laperierre N, Mehta M, Curran W (2006) Phase III trial of chemotherapy plus radiotherapy compared with radiotherapy alone for pure and mixed anaplastic oligodendroglioma: intergroup Radiation Therapy Oncology Group Trial 9402. J Clin Oncol 24:2707-2714

15. Wick W, Hartmann C, Engel C, Stoffels M, Felsberg J, Stockhammer F, Sabel MC, Koeppen S, Ketter R, Meyermann R, Rapp M, Meisner C, Kortmann RD, Pietsch T, Wiestler OD, Ernemann U, Bamberg M, Reifenberger G, von Deimling A, Weller M (2009) NOA-04 randomized phase III trial of sequential radiochemotherapy of anaplastic glioma with procarbazine, lomustine, and vincristine or temozolomide. J Clin Oncol 27:5874-5880

16. Bauman GS, Ino Y, Ueki K, Zlatescu MC, Fisher BJ, Macdonald DR, Stitt L, Louis DN, Cairncross JG (2000) Allelic loss of chromosome $1 \mathrm{p}$ and radiotherapy plus chemotherapy in patients with oligodendrogliomas. Int J Radiat Oncol Biol Phys 48: 825-830

17. Brandes AA, Tosoni A, Cavallo G, Reni M, Franceschi E, Bonaldi L, Bertorelle R, Gardiman M, Ghimenton C, Iuzzolino P, Pession A, Blatt V, Ermani M (2006) Correlations between O6methylguanine DNA methyltransferase promoter methylation status, $1 \mathrm{p}$ and $19 \mathrm{q}$ deletions, and response to temozolomide in anaplastic and recurrent oligodendroglioma: a prospective GICNO study. J Clin Oncol 24:4746-4753

18. Kouwenhoven MC, Kros JM, French PJ, Biemond-ter SE, Graveland WJ, Taphoorn MJ, Brandes AA, van den Bent MJ (2006) $1 \mathrm{p} / 19 \mathrm{q}$ loss within oligodendroglioma is predictive for response to first line temozolomide but not to salvage treatment. Eur J Cancer 42:2499-2503

19. Yan H, Parsons DW, Jin G, McLendon R, Rasheed BA, Yuan W, Kos I, Batinic-Haberle I, Jones S, Riggins GJ, Friedman H, Friedman A, Reardon D, Herndon J, Kinzler KW, Velculescu VE, 
Vogelstein B, Bigner DD (2009) IDH1 and IDH2 mutations in gliomas. N Engl J Med 360:765-773

20. Sanson M, Marie Y, Paris S, Idbaih A, Laffaire J, Ducray F, El HS, Boisselier B, Mokhtari K, Hoang-Xuan K, Delattre JY (2009) Isocitrate dehydrogenase 1 codon 132 mutation is an important prognostic biomarker in gliomas. J Clin Oncol 27:4150-4154

21. Thompson CB (2009) Metabolic enzymes as oncogenes or tumor suppressors. N Engl J Med 360:813-815

22. Zhao S, Lin $Y, X u$ W, Jiang W, Zha Z, Wang P, Yu W, Li Z, Gong L, Peng Y, Ding J, Lei Q, Guan KL, Xiong Y (2009) Glioma-derived mutations in IDH1 dominantly inhibit IDH1 catalytic activity and induce HIF-1alpha. Science 324:261-265

23. Dang L, White DW, Gross S, Bennett BD, Bittinger MA, Driggers EM, Fantin VR, Jang HG, Jin S, Keenan MC, Marks KM, Prins RM, Ward PS, Yen KE, Liau LM, Rabinowitz JD, Cantley LC, Thompson CB, Vander HM, Su SM (2009) Cancer-associated IDH1 mutations produce 2-hydroxyglutarate. Nature 462:739-744

24. Cairncross G, Berkey B, Shaw E, Jenkins R, Scheithauer B, Brachman D, Buckner J, Fink K, Souhami L, Laperierre N, Mehta M, Curran W (2006) Phase III trial of chemotherapy plus radiotherapy compared with radiotherapy alone for pure and mixed anaplastic oligodendroglioma: intergroup Radiation Therapy Oncology Group Trial 9402. J Clin Oncol 24:2707-2714

25. Li S, Yan C, Huang L, Qiu X, Wang Z, Jiang T (2012) Molecular prognostic factors of anaplastic oligodendroglial tumors and its relationship: a single institutional review of 77 patients from China. Neuro Oncol 14:109-116

26. Watanabe T, Nobusawa S, Kleihues P, Ohgaki H (2009) IDH1 mutations are early events in the development of astrocytomas and oligodendrogliomas. Am J Pathol 174:1149-1153

27. Snuderl M, Eichler AF, Ligon KL, Vu QU, Silver M, Betensky RA, Ligon AH, Wen PY, Louis DN, Iafrate AJ (2009) Polysomy for chromosomes 1 and 19 predicts earlier recurrence in anaplastic oligodendrogliomas with concurrent $1 \mathrm{p} / 19 \mathrm{q}$ loss. Clin Cancer Res 15:6430-6437

28. Wiens AL, Cheng L, Bertsch EC, Johnson KA, Zhang S, Hattab EM (2012) Polysomy of chromosomes 1 and/or 19 is common and associated with less favorable clinical outcome in oligodendrogliomas: fluorescent in situ hybridization analysis of 84 consecutive cases. J Neuropathol Exp Neurol 71:618-624 